\begin{tabular}{c|c|c|c|} 
SELECCIONES MATEMÁTICAS \\
Universidad Nacional de Trujillo \\
ISSN: $2411-1783$ (Online) \\
2021; Vol. 8(1): $125-130$.
\end{tabular}

\title{
Rotational flows over obstacles in the forced Korteweg-de Vries framework
}

\author{
Marcelo V. Flamarion $『$
}

Received, Mar. 27, 2021

Accepted, May. 26, 2021

How to cite this article:

Flamarion MV. Rotational flows over obstacles in the forced Korteweg-de Vries framework. Selecciones Matemáticas. 2021;8(1):125-130. http://dx.doi.org/10.17268/sel.mat.2021.01.12

\begin{abstract}
In this work we investigate rotational waves resonantly excited by a submerged obstacle in a sheared shallow water channel with constant vorticity. In the weakly nonlinear, weakly dispersive regime, the problem is formulated in the forced Korteweg-de Vries framework. We compute the solution of the initial value problem for this equation numerically using a Fourier pseudospectral method with integrating factor. The water surface is initially taken at rest, and once the current is turned on, waves are immediately generated in the free surface. We identify the main effects of sheared current in the generated waves such as rotational solitary waves propagating upstream and sharp crested waves being generated.
\end{abstract}

Keywords . Gravity waves, Solitary waves, KdV equation, Shear flow.

1. Introduction. In order to investigate problems in hydrodynamics, we usually need an external force to model the propagation of a water wave, i.e., a mechanism that produce waves [10]. Physically, the external force usually models two types of problems: waves generated by a moving disturbance in the bottom of a channel or a distribution of pressure moving along the free surface. There are several application problems in these scenarios, we mention atmospheric flows encountering topographic obstacles, flow of water over rocks [2], ship wakes and ocean waves generated by storms [8].

When the flow is irrotational, the waves generated due to a current-topography interaction are governed by two parameters, namely, the amplitude of the external force and the Froude number defined as

$$
F=\frac{U_{0}}{\sqrt{g h_{0}}}
$$

where, $U_{0}$ is the velocity of the uniform stream, $g$ is the acceleration of gravity and $h_{0}$ is the undisturbed depth of a shallow water channel. In the weakly nonlinear, weakly dispersive regime the forced Kortewegde Vries $(\mathrm{fKdV})$ can be used as model to study near-critical flows $(F \approx 1)$ produced by external forces with small amplitudes. Several authors have studied flow over obstacles in the fKdV framemork. This topic is broad and therefore it is hard to give an overview of contributions. For the interested reader, we mention a few articles and references therein from which the bibliography may be useful. The first study reported using the fKdV as a model to study flow over obstacles was done by $\mathrm{Wu}$ and $\mathrm{Wu}[13]$, later in [1, 5, 12, 9], and more recently by Ermakov and Stepanyants [3].

In the near-resonant regime, the generated waves have complex patterns which propagates upstream and downstream away from where the obstacle is located. These flows are undesirable since the generated waves may cause problems in applications, such as the erosion of waterway banks and energy loss through wave drag on a ship. In the supercritical regime $(F>1)$, the flow is mainly characterized by the formation of a dominant elevation wave stationed exactly above the obstacle, while a smaller transient wave train moves downstream. The steady state is reached when the transient wave train sheds. In subcritical case $(F<1)$, the flow is featured by a periodic wave train propagating downstream and a small transient

*UFRPE/Rural Federal University of Pernambuco, UACSA/Unidade Acadêmica do Cabo de Santo Agostinho, BR 101 Sul, 5225 , 54503-900, Ponte dos Carvalhos, Cabo de Santo Agostinho, Pernambuco, Brazil. (marcelo. flamarion@ufrpe.br). 
wave moving upstream. The steady state corresponds to an upstream surface elevation with a downstream periodic wave train.

The Korteweg-de Vries in the presence of a background flow was first introduced by Johnson and Freeman [7]. However, the authors did not consider the presence of an external force in their formulation. Only recently, a nonuniform bottom and a moving distribution of pressure was taken into account the fKdV framework [4]. Using asymptotic analysis for the full Euler equations, Flamarion et al. [4] deduced a fKdVtype equation that can be used to model rotational flows over obstacles. In this work, the authors showed that mathematically the effects of pressure and topography are the same, and used the obtained fKdV to validate a numerical method for the full Euler equation.

In this work we investigate numerically the rotational flow over an obstacle in a shallow water channel with constant vorticity in the fKdV framework. We focus on the effects of the vorticity on the generated waves. To the best of our knowledge there are no articles on rotational generated waves in the fKdV framework. From our numerical experiments, we identify that in the critical regime rotational $s e c h^{2}$-type solitary waves propagate upstream, and this waves become sharper as the current gets stronger in the bottom. In the supercritical regime, an elevation wave arise exactly over the obstacle and its amplitude increases when the current is strong in the bottom of the channel. Moreover, sharp crested waves are observed in the wave train that propagates downstream in the subcritical regime.

This article is organized as follows. In section 2 we present the mathematical formulation of the rotational $\mathrm{fKdV}$ equation. The numerical methods are presented in section 3 , the numerical results in section 4 and the conclusion in section 5.

2. The rotational forced Korteweg-de Vries equation. We consider a two-dimensional incompressible flow of an inviscid fluid with constant density in a sheared shallow water channel with constant vorticity in the presence of gravity force and a submerged obstacle.

In the weakly nonlinear regime, the dimensionless fKdV equation

$$
-2 I_{31} \zeta_{t}-2 I_{31} f \zeta_{x}+3 I_{41} \zeta \zeta_{x}+J_{1} \zeta_{x x x}=h_{x}(x),
$$

can be used as a model to describe the flow over disturbances with small amplitudes [4]. Here, $\zeta(x, t)$ is the free-surface displacement over the undisturbed surface and $h$ is the submerged obstacle. The coefficients in (2.1) are given by the formulas

$$
I_{31}=\frac{\Omega+2 \gamma(\Omega)}{2 \gamma(\Omega)^{2}(\Omega+\gamma(\Omega))^{2}}, \quad I_{41}=\frac{1}{3} \frac{\Omega^{2}+3 \Omega \gamma(\Omega)+3 \gamma(\Omega)^{2}}{\gamma(\Omega)^{3}(\Omega+\gamma(\Omega))^{3}}, \quad J_{1}=\frac{1}{3 \gamma(\Omega)^{3}} .
$$

where

$$
\gamma(\Omega)=-\frac{\Omega}{2}+\frac{\sqrt{\Omega^{2}+4}}{2}
$$

$\Omega$ is the vorticity parameter and

$$
U(y)=\Omega y+\gamma(\Omega)+\epsilon f,
$$

is the sheared current of constant vorticity $(\omega=-\Omega), f$ is a constant, and $\epsilon$ is a small positive parameter. The $\mathrm{fKdV}$ is obtained through asymptotic analysis from the dimensionless full Euler equations. In the full Euler model, the bottom of the channel is $y=-1$, and $y=0$ is the undisturbed surface. The flow is defined as supercritical, subcritical or near-critical depending on whether $f>0, f<0$ or $f \approx 0$.

The coefficients of (2.1) are functions of the vorticity parameter. We point out that the nonlinearity term never vanishes, and it increases as the absolute value of $\Omega$ increases. Besides, the dispersive term decays to zero as $\Omega \rightarrow-\infty$. Thus, in this limit the $\mathrm{fKdV}$ equation (2.1) approaches the Burgers equation, and the waves may break. These coefficients as a function of $\Omega$ are depicted in Figure 2.1.

When the bottom is flat $\left(h_{x}=0\right)$ a traveling solitary wave solution for $(2.1)$ is

$$
\zeta(x, t)=\operatorname{Asech}^{2}(k(x-c t)), \quad \text { where } c=f-\frac{2 J_{1}}{I_{31}} k^{2}, \quad A=\frac{4 J_{1}}{I_{41}} k^{2},
$$

and the unforced linearized equation $(2.1)$ is

$$
\zeta_{t}+f \zeta_{x}-\frac{J_{1}}{2 I_{31}} \zeta_{x x x}=0 .
$$

Therefore, linear waves with wavelength $\lambda=2 \pi / k$ propagates with phase speed

$$
c(k)=f+\frac{J_{1}}{2 I_{31}} k^{2} .
$$



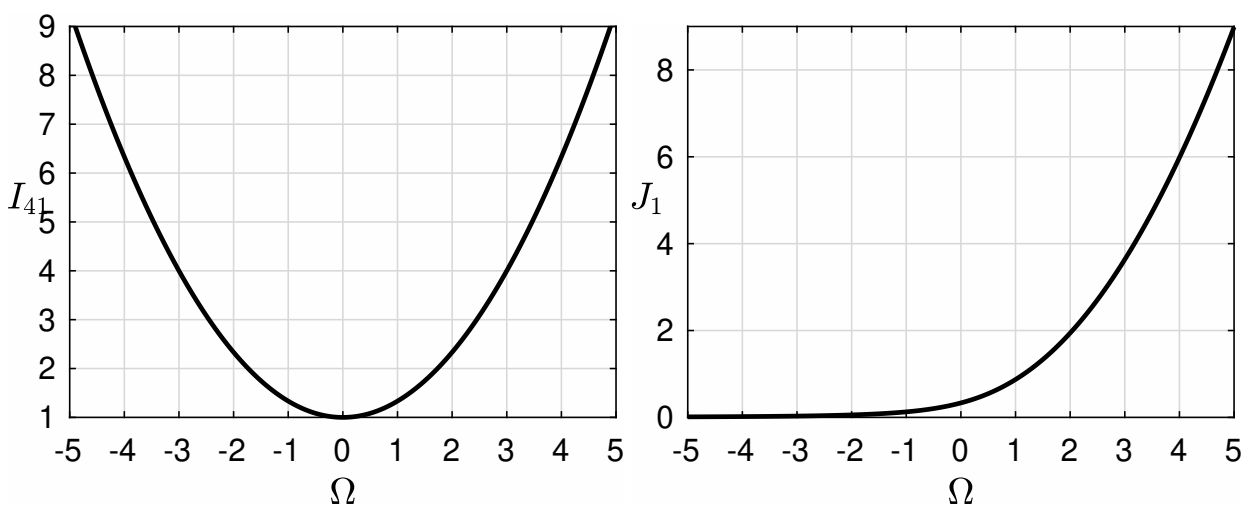

Figure 2.1: Left: the coefficient $I_{41}$ as a function of $\Omega$. Right: the coefficient $J_{1}$ as a function of $\Omega$.

In particular, steady linear waves only exist if

$$
k=\sqrt{-\frac{2 I_{31}}{J_{1}} f} .
$$

Since $I_{31}, J_{1}>0$, steady waves only exist when the flow is subcritical $(f<0)$. Figure 2.2 displays the linear phase speed for $f=0$ and different values of $\Omega$. It is possible to see that when the current is weaker at the bottom $(\Omega>0)$, these linear waves travel slower. Furthermore, in the supercritical regime, linear waves always travel downstream. However, in the subcritical regime waves can propagate downstream as well as upstream depending on their wavelength.

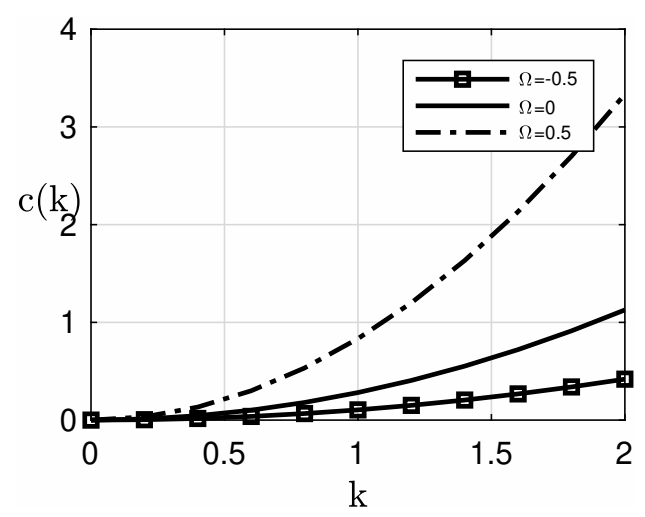

Figure 2.2: The linear phase speed of (2.5) for $f=0$ and different values of $\Omega$.

3. Numerical methods. The fKdV equation (2.1) is solved using a Fourier pseudospectral method with an integrating factor. It avoids numerical instabilities due to the higher-order derivative term. We consider a periodic computational domain $[-L, L]$ with a uniform grid with $N$ points and step $\Delta x=L / N$. The derivatives in $x$ are performed spectrally using the Fast Fourier Transform (FFT) [11]. Moreover, the time advance is computed through the classical Runge-Kutta fourth-order method (RK4) with time step $\Delta t$. The sensitivity of a similar numerical method with respect to $N$ and $\Delta x$ was tested in [4]. The authors showed that the solution is accurately captured by different discretizations. Thus, for convenience, we set the following parameters: $L=1000, N=2^{13}$, and $\Delta t=0.01$. The initial data is always taken at rest $(\zeta(x, 0)=0)$ and the bottom topography is modelled by the localised obstacle

$$
h(x)=\frac{e^{-x^{2}}}{\sqrt{\pi}} .
$$

Although $h$ is not periodic, $h$ decays exponentially to zero as $|x| \rightarrow \infty$ therefore, for large values of $L$, we can approximate the boundary conditions by periodic conditions.

4. Numerical results. We start our discussion considering the critical regime. In the absence of vorticity, it is well known that solitary waves are generated propagating upstream, and a wave train downstream 
[13]. When the current is no longer constant, we observe that rotational solitary waves are generated periodically upstream and an oscillatory wave train downstream. We compute the amplitude of the leading solitary wave propagating upstream and compare it to the $s e c h^{2}$-type solitary waves defined in (2.4) and find that they agree very well. A particular case is depicted in Figure 4.1. Although the pattern of the generated waves are qualitatively similar to the irrotational problem, we point out a few differences. First, when the current is stronger at the bottom of the channel $(\Omega<0)$, waves are generated more frenetically upstream as well as downstream, and their crests are sharper. On the other hand, when the current is stronger close to the surface, waves are slowly generated and their crest become smoother. These features can be seen with more details in Figure 4.2.

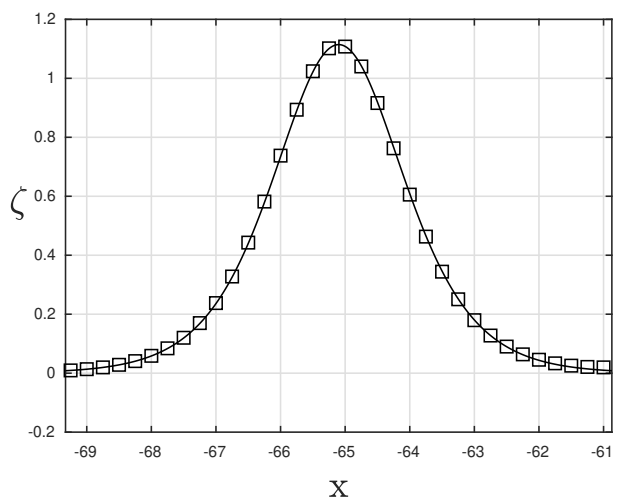

Figure 4.1: Comparison between the lead with upstream when $\Omega=0.5$ and the solitary wave defined in (2.4). Solid line represents the leading wave upstream, and the squares represent the solitary wave given by the formula (2.4).
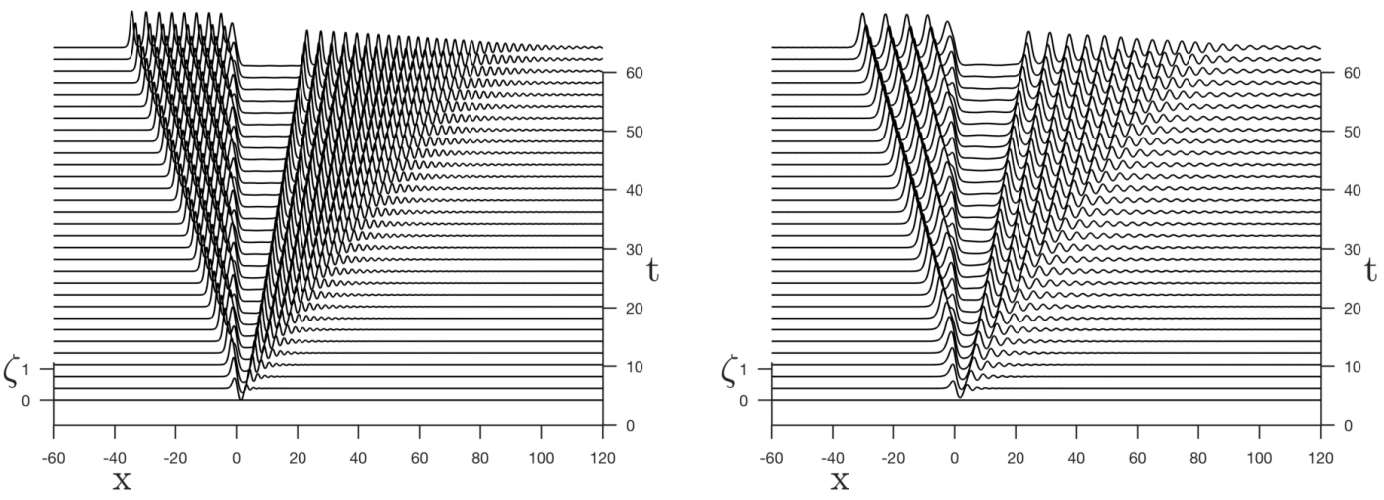

Figure 4.2: Left: generated waves in the critical case for $\Omega=-0.5$. Right: generated waves in the critical case for $\Omega=0.5$.

Now we consider the supercritical regime. In this case we see that the amplitude of the resonant wave with the topography increases when the shear current is stronger at the bottom. Besides, these waves become crested sharper. The steady state is reached when the transient propagating downstream shed. Figure 4.3 (left) displays a typical solution of equation (2.1) in the supercritical regime. As we can see in Figure 4.3 (right) the amplitude of the steady wave decreases with $\Omega$. It is as the problem somehow becomes more linear for large values of $\Omega$.

Lastly, we analyse the subcritical regime. In this case, waves are generated upstream and a train of waves downstream. When the current is stronger at the bottom, waves propagate faster and their crest are sharper. The corresponding steady solution is an elevation surface on the left of the topography and a steady wave train downstream.

These results help us understand some important features in the full model. For instance, Flamarion et al. [4] found numerical evidences that the generated waves due to a current-topography interaction may break at early times when $\Omega$ is negative. Although the fKV model cannot capture wave breaking, we find that in this case, the generated the waves become sharp crested, which indicates that they may break in the full model. 

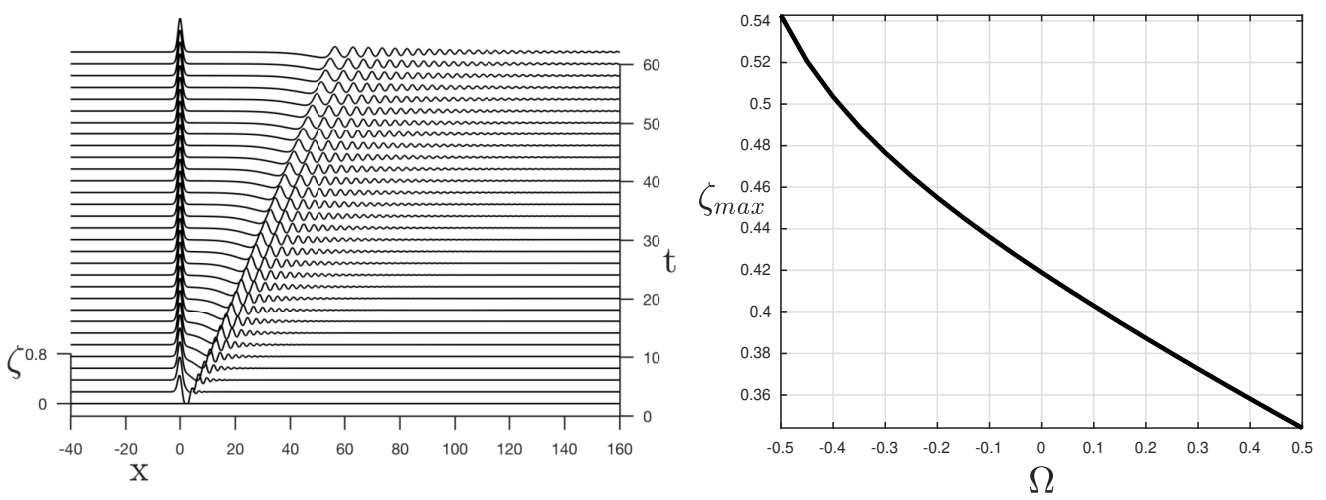

Figure 4.3: Left: Typical supercritical generated wave for $f=0.75$ and $\Omega=-0.5$. Right: The amplitude of supercritical waves with $f=0.75$ as a function of $\Omega$.
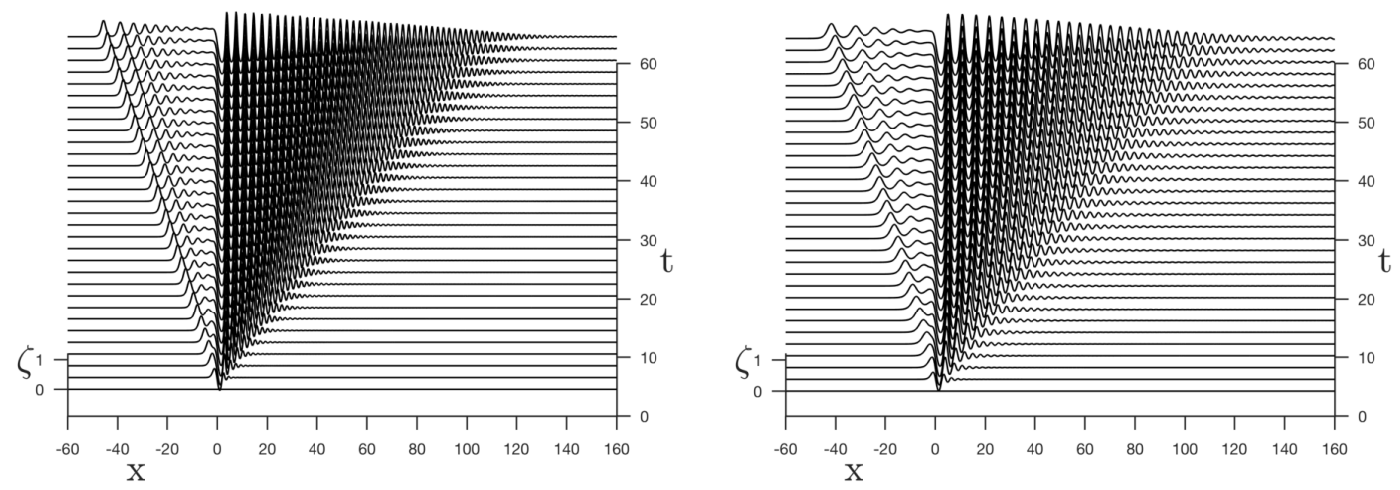

Figure 4.4: Left: generated waves in the subcritical case for $f=-0.5$ and $\Omega=-0.5$. Right: generated waves in the subcritical case for $f=-0.5$ and $\Omega=0.5$.

5. Conclusions. In this paper we have investigated rotational flows over a submerged obstacle. The problem was formulated using the rotational $\mathrm{fKdV}$ framework. Through a pseudospectral numerical method, we investigate how the sheared flow affects the generated waves. Qualitatively, the flow is still governed by a the amplitude of the obstacle and the small the Froude number. Although the patterns of the generated waves are similar to the ones observed in the irrotational problem, some aspects such as the generation of rotational solitary waves, sharp crested waves arise. In particular, the formation of sharp crested waves indicate that solution of the full Euler model may break at early times. In the critical regime we compared the generated upstream waves with $\operatorname{sech}^{2}$-type solitary waves and found that they agreed well. In the supercritical regime, when the current is stronger in the bottom of the channel, the amplitude of the elevation wave over the obstacle is higher. Besides, in the subcritical regime the wave train that propagates downstream becomes sharp crested when the current is stronger in the bottom of the channel.

6. Acknowledgements. The author is grateful to IMPA-National Institute of Pure and Applied Mathematics for the research support provided during the Summer Program of 2020 and to Federal University of Paraná for the visit to the Department of Mathematics.

ORCID and License

Marcelo V. Flamarion https://orcid.org/0000-0001-5637-7454

This work is licensed under the Creative Commons - Attribution 4.0 International (CC BY 4.0)

\section{References}

[1] Akylas TR. On the excitation of long nonlinear water waves by a moving pressure distributions. J Fluid Mech. 1984; 141:455466. DOI: $10.1017 / \mathrm{S} 0022112084000926$. 
[2] Baines P. Topographic effects in stratified flows. Cambridge: Cambridge University Press; 1995.

[3] Ermakov E, Stepanyants Y. Soliton interaction with external forcing within the Korteweg-de Vries equation. Chaos. 2019; 29:1-14. DOI: $10.1063 / 1.5063561$.

[4] Flamarion MV, Milewski PA, Nachbin A. Rotational waves generated by current-topography interaction. Stud Appl Math. 2019; 142: 433-464. DOI: 10.1111/sapm.12253.

[5] Grimshaw R, Smyth N. Resonant flow of a stratified fluid over topography in water of finite depth. J. Fluid Mech. 1986; 169:235-276. DOI: 10.1017/S002211208600071X.

[6] Grimshaw R, Pelinovsky E, Tian X. Interaction of a solitary wave with an external force. Physica D. 1994; 77:405-433. DOI: 10.1002/sapm1996973235.

[7] Johnson RS, Freeman NC. Shallow water waves on shear flows. J. Fluid Mech. 1970; 42:401-409. DOI: $10.1017 /$ S0022112070001349.

[8] Johnson RS. Models for the formation of a critical layer in water wave propagation. Phil. Trans. R. Soc. A. 2012; 370:16381660. DOI: 10.1098/rsta.2011.0456.

[9] Milewski PA. The Forced Korteweg-de Vries equation as a model for waves generated by topography. CUBO A mathematical Journal. 2004; 6:33-51.

[10] Pratt LJ. On nonlinear flow with multiple obstructions. J. Atmos. Sci. 1984; 41:1214-1225. DOI: 10.1175/1520-0469.

[11] Trefethen LN. Spectral Methods in MATLAB. Philadelphia: SIAM; 2001.

[12] Wu TY. Generation of upstream advancing solitons by moving disturbances. J Fluid Mech. 1987; 184:75-99. DOI: $10.1017 /$ S0022112087002817.

[13] Wu DM, Wu TY. Three-dimensional nonlinear long waves due to moving surface pressure. In: Proc. 14th. Symp. on Naval Hydrodynamics. 1982; Washington, DC. Nat. Acad. Sci., 1982; pp103-125. 